\title{
Efficient Transmitter and Receiver Designs for SC-FDMA Based Heterogeneous Networks
}

\author{
${ }^{1}$ Daniel Castanheira, ${ }^{1}$ Adão Silva, ${ }^{2}$ Rui Dinis, ${ }^{1}$ Atílio Gameiro \\ ${ }^{1}$ DETI, Instituto de Telecomunicações, University of Aveiro, Portugal \\ ${ }^{2}$ Instituto de Telecomunicações, Faculdade de Ciências e Tecnologia, Univ. Nova de Lisboa, Portugal \\ Emails: dcastanheira@av.it.pt, asilva@av.it.pt, rdinis@lx.it.pt, amg@ua.pt
}

\begin{abstract}
In this manuscript, we consider the uplink of a single carrier frequency division multiple access (SC-FDMA) based system, where a set of small-cells coexist with a macro-cell using the same spectrum. To deal with both the inter-carrier interference (ICI) and inter-system interference we combine interference alignment (IA) precoding at the small-cell transmitters with iterative decision feedback equalization at the macroreceiver. The transmitter and receiver design is performed jointly, by considering both full-coordination and limited inter-system information exchange between macro and small-cells. At the small cells side, we assume that the access points are connected through a limited capacity backhaul network to a central unit, where the separation of a quantized version of the small-cell signals is performed. For this case the iterative feedback equalizer is designed by explicitly taking into account the impact of signal quantization. Moreover, a semi-analytical approach for the performance of the proposed schemes is provided. The results show that the proposed schemes are robust to both inter-system and inter-carrier interferences and thus are able to efficiently separate the macro and small cells spatial streams under limited information exchange.
\end{abstract}

Index Terms-Heterogeneous Networks, SC-FDMA, Interference Alignment, IB-DFE, Quantization

\section{INTRODUCTION}

To cope with the increasing demand for wireless services the operators are considering the deployment of small-cells within the boundaries of the existing macro-cells [1]. Beyond the capacity improvements, small-cells offer other advantages for the operators: they are easier to deploy, less expensive and more energy efficient, just to mention a few benefits. Nevertheless, due to the expected extensive deployment of small-cells and costs involved in the acquisition of new spectrum licenses the two systems are likely to coexist within the same spectrum. This requires the development of efficient interference management techniques because if not carefully designed the small-cells signals may generate harmful interference on the macro-cell [2]. As the macro-cell is the licensee (the owner of the spectrum license), to deal with the interference problem small-cells must reuse the unused macro-cell resources. These opportunities may be in time, frequency or even in the space dimension. When it comes to the latter the macro-cell terminals may not use all the spatial dimensions due to physical constraints that do not allow a number of antennas as large as in the base station (BS) or as pointed out in [3], where optimal power allocation may lead to unused spatial resources. In both cases, this leads to unused spatial dimensions at the macro-cell that can be exploited by the small-cell terminals. Active use of the spatial domain through multiple antenna techniques is of outmost importance to minimize the interference levels in a heterogeneous network ${ }^{1}$ environment. Interference alignment is a precoding technique that is able to achieve the maximum degrees of freedom of the interference channel, in a variety of settings [5][6]. In some cases (e.g., constant channels) this may require long symbol extensions, either across time or across frequency [7]. IA works by dividing the receiver space in two parts, one for interference and the other for the intended signal. By using IA, all inter-user interference is aligned in the interference space, restricting in that way the interference subspace dimensions. Closed form IA solutions are only available for some specific cases [8]. For the other cases, iterative methods may be used [8]-[10]. In [8], two iterative algorithms were proposed that take advantage of reciprocity in wireless networks to achieve IA, with only local channel knowledge at each node. A convergent alternating minimization approach to IA was proposed in [9]. In [10] the authors presented two algorithms that optimize the precoding subspaces by maximizing the data rate performance while maintaining the achievable degrees of freedom.

IA techniques were recently proposed to mitigate the interference of small-cell user terminals (UTs) towards the macrocell BS [11], [12] and [13]. In [11] the authors proposed a new interference alignment scheme that successively creates transmit beamforming vectors for the small-cell terminals and for the macro BS assuming that they have different number of transmit antennas. A scheme that takes advantage of the orthogonal frequency division multiplexing (OFDM) cyclic prefix for interference alignment was proposed in [12]. The work in [13] studied several IA techniques with different levels of inter-system information sharing: a coordinated IA with large information sharing requirements, a static IA and an uncoordinated IA without any information sharing. The problem of IA precoded, under limited information exchange, for narrowband heterogeneous based networks, has been analytically tackled in [14].

In [15] the authors presented the first experimental study of IA in measured multiple-input multiple-output OFDM (MIMO-OFDM) interference channels. Due to the high peak-

\footnotetext{
${ }^{1}$ We use the expression heterogeneous networks to designate a system employing macro and small cells with loose coupling. In the loose coupling architecture, the networks are deployed as a complement to each other and they are operated by the service provider individually and independently [4].
} 
to-average power ratio (PAPR) OFDM is mainly used for the downlink. On the other hand, SC-FDMA is a promising solution technique for high data rate uplink communications in future cellular systems. The principal advantage of SC-FDMA is the PAPR, which is lower than that of OFDMA [16], [17]. SC-FDMA was adopted for the uplink, as a multiple access scheme, of the current long-term evolution (LTE) cellular system [18]. Single-carrier frequency domain equalization (SC-FDE) is widely recognized as an excellent alternative to OFDM, especially for the uplink of broadband wireless systems [19], [20]. Conventional SC-FDE schemes employ a linear FDE optimized under the minimum mean square error (MMSE) criterion. However, the residual interference levels might still be too high, leading to performance that is still several $\mathrm{dB}$ from the matched filter bound (MFB) [21]. For this reason, there has been significant interest in the design of nonlinear FDE in general and decision feedback FDE in particular, with the iterative block decision feedback equalizer (IB-DFE) being the most promising nonlinear FDE [21]. IBDFE was originally proposed in [22] and was extended for a wide range of scenarios in the last years, ranging from diversity scenarios, multiplexing MIMO and multicarrier code division multiple access (MC-CDMA) systems, among many others [23], [24].

In this manuscript we combine IA precoding at the smallcell transmitters with IB-DFE based receivers at the macrocell. The main motivation is that, IA based schemes are quite efficient to deal with inter-system interference. However, IA is not specifically designed to deal with the ICI that arises in SCFDMA based systems. Thus, in the context of heterogeneous systems, this combination allows us to design the transmitters and receivers to cope with both inter-system and inter-carrier interferences, achieving the high order diversity offered by the MIMO SC-FDMA systems and thus efficiently separate the macro and small cell spatial streams. To the best of our knowledge the joint design of IB-DFE based receivers and IA precoders for heterogeneous systems has not been addressed in the literature. In a first approach, the transmitters and macroreceiver are jointly optimized by considering full coordination between the two systems and then a limited inter-system information exchange approach is derived. For the optimized approach, it is demonstrated that the small-cells must align their transmission along the null-space of the macro-channel. The amount of information required to inform the small cells about the macro-channel would be very high, resulting in poor system efficiency and high cooperation delays. To limited these problems for the limited inter-system information approach the macro-cell forwards to the small-cells a quantized version of the macro-cell channel. This information is used by the small-cell UTs to align the interference along the null-space of the quantized macro-channel. At the small cells, we consider that the access points (APs) are connected through a limited capacity backhaul network to a central unit (CU), where the separation of a quantized version of the small-cell signals is performed. An efficient iterative feedback equalizer, based on the IB-DFE principles, is designed by explicitly taking into account the signal quantization errors.

The proposed receiver structures are explicitly designed

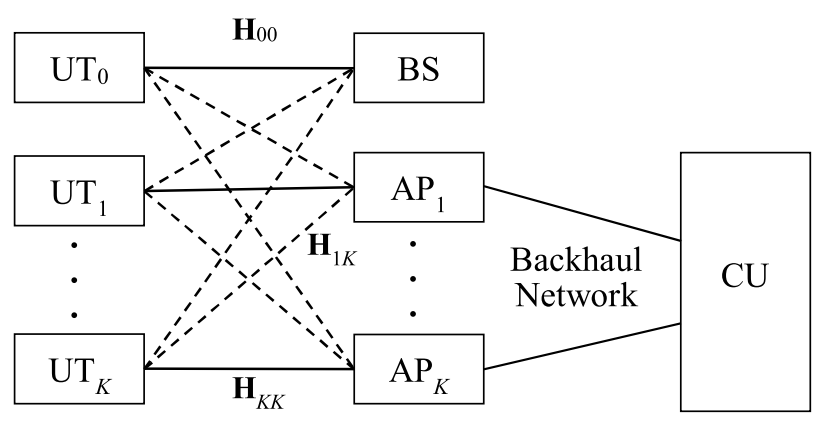

Fig. 1. Block diagram of the considered system.

taking into account inter-system and residual inter-carrier interferences, allowing an efficient co-existence of both systems. Furthermore, we propose a simple, yet accurate semianalytical approach for obtaining the performance of the proposed receiver structures.

This manuscript is organized as follows: Section II presents the system model. In section III, we start by jointly designing the small-cell precoders and macro equalizer and then we propose an efficient approach to reduce the inter-system information exchange requirements. In this section, we also design the small-cell receiver by taking into account the impact of the signal quantization. Section IV presents the main simulation results. The conclusions will be drawn in section V.

Notations: Boldface capital letters denote matrices and boldface lowercase letters denote column vectors. The operations $(.)^{H}$ and $\operatorname{tr}($.$) represent the Hermitian transpose and the trace$ of a matrix. $\left\{\alpha_{l}\right\}_{l=1}^{L}$ represents an $L$ length sequence, $\mathbf{I}_{N}$ the identity matrix with size $N \times N$ and $\mathcal{N}(\mathbf{A})$ a matrix whose columns span the null space of $\mathbf{A} . \mathbf{c}_{n, k}^{l, i}(p)$ denotes element $p$ of vector $\mathbf{c}_{n, k}^{l, i}$, where $n, k, l$ and $i$ are the receiver, transmitter, subcarrier, and iteration indices, respectively.

\section{System MOdEL}

We consider a scenario with a set of $K$ small-cells overlaid by a macro-cell as shown in Fig. 1 . We assume that both the macro and small-cells are operating in time division duplex (TDD) mode. The $N$ small-cell APs are connected through a limited backhaul network to a CU. The uplink for both the macro and small-cells uses SC-FDMA as the access technique, with $L$ available subcarriers. In the following, the macro-cell UT is denominated by $\mathrm{UT}_{0}$ and the UT (AP) in small-cell $k$ by UT (AP) $k$. The index $l$ is used to denote a given subcarrier ${ }^{2}$ of the $L$ available.

\section{A. Macro-Cell}

The macro-cell has a BS and an associated UT. The number of antennas at the BS and the $\mathrm{UT}$ is $T_{0}$ and $M_{0}$, respectively. In the next sections, it is assumed that $T_{0}>M_{0}$ since due ${ }^{2}$ Although we are considering single-carrier modulations, we can still use
the subcarrier concept, which is associated to the different samples of the DFT of the time-domain data block. 
to physical constraints at the transceivers it is likely that the number of antennas at the user terminal will be lower than the number of antennas at the BS. The received frequency-domain signal (i.e., after cyclic prefix removal and discrete Fourier transform (DFT) operation) at the BS and the $l$ th subcarrier is given by

$$
\mathbf{y}_{B S}^{l}=\underbrace{\mathbf{H}_{00}^{l} \mathbf{x}_{0}^{l}}_{\text {Desired Signal }}+\underbrace{\sum_{k=1}^{K} \mathbf{H}_{0 k}^{l} \mathbf{x}_{k}^{l}}_{\text {Inter-System Interference }}+\underbrace{\mathbf{n}_{0}^{l}}_{\text {Noise }}
$$

where $\mathbf{H}_{00}^{l} \in \mathbb{C}^{T_{0} \times M_{0}}$ denotes the macro-cell channel, $\mathbf{H}_{0 k}^{l} \in$ $\mathbb{C}^{T_{0} \times M_{k}}$ the channel from UT $k$ to the BS, $\mathbf{x}_{0}^{l}$ the macrocell transmit signal on $l$ th subcarrier, $\left\{\mathbf{x}_{k}^{l}\right\}_{k=1}^{K}$ the small-cells transmitted signal and $\mathbf{n}_{0}^{l}$ the white Gaussian noise, with variance $\sigma_{n}^{2}$. The macro-BS has channel state information (CSI) of channel $\mathbf{H}_{00}^{l}$ and channel $\mathbf{H}_{0 k}^{l}$ is known at UT $k$. This CSI may be acquired by listening to the pilots and/or training sequences broadcasted by the macro-UT (macro-BS), since the macro cell and small cells mode of operation is TDD.

The macro UT uses all available spatial resources for data transmission, i.e., $P_{0}=M_{0}$ data streams are transmitted at each subcarrier. Let us denote by $\left\{\mathbf{d}_{0}^{m}\right\}_{m=1}^{L}$ the macro-cell data symbols and by $\mathbf{d}_{0}^{m}(p)$ the corresponding $p$ th element of vector $\mathbf{d}_{0}^{m}$ (with $\mathbb{E}\left[\left|\mathbf{d}_{0}^{m}(p)\right|^{2}\right]=\Sigma_{0}^{2}$ ). The data symbols are drawn from a $M$-QAM constellation. To form the transmit signal, the macro UT applies DFT to the sequence $\left\{\mathbf{d}_{0}^{m}(p)\right\}_{m=1}^{L}$

$$
\left\{\mathbf{x}_{0}^{l}(p)\right\}_{l=1}^{L}=\operatorname{DFT}\left(\left\{\mathbf{d}_{0}^{m}(p)\right\}_{m=1}^{L}\right)
$$

To normalize the notation, for the macro and small-cells we denote by $\left\{\mathbf{s}_{0}^{l}(p)\right\}_{l=1}^{L}$ the DFT of the data symbols $\left\{\mathbf{d}_{0}^{m}(p)\right\}_{m=1}^{L}$. For the macro-cell, the sequence $\left\{\mathbf{s}_{0}^{l}(p)\right\}_{l=1}^{L}$ is the transmitted signal but for the small-cells we apply a precoder to this sequence before transmission.

\section{B. Small-Cells}

Each small-cell has one AP and an associated UT. AP $k$ has $T_{k}$ antennas and UT $k M_{k}$ antennas. The received signal in frequency domain at $\operatorname{AP} n$ and subcarrier $l$ is

$$
\mathbf{y}_{n}^{l}=\underbrace{\mathbf{H}_{n n}^{l} \mathbf{x}_{n}^{l}}_{\text {Desired Signal }}+\underbrace{\sum_{k=0, k \neq n}^{K} \mathbf{H}_{n k}^{l} \mathbf{x}_{k}^{l}}_{\text {Inter-System/User Interference }}+\underbrace{\mathbf{n}_{n}^{l}}_{\text {Noise }}
$$

where $\mathbf{H}_{n k}^{l} \in \mathbb{C}^{T_{n} \times M_{k}}$ denotes the channel from UT $k$ to the $\mathrm{AP} n$ at subcarrier $l$ and $\mathbf{n}_{n}^{l}$ white Gaussian noise with variance $\sigma_{n}^{2}$. Small-cell UT $k$ has CSI of channel $\mathbf{H}_{0 k}^{l}$, which may be acquired by taking advantage of the channel reciprocity properties for TDD systems. The transmit signal at UT $k$ is obtained through the linear map $\mathbf{V}_{k}^{l} \in \mathbb{C}^{M_{k} \times P_{k}}$

$$
\mathbf{x}_{k}^{l}=\mathbf{V}_{k}^{l} \mathbf{s}_{k}^{l}
$$

where $\mathbf{s}_{k}^{l}=\left[\mathbf{s}_{k}^{l}(1), \ldots, \mathbf{s}_{k}^{l}(p), \ldots, \mathbf{s}_{k}^{l}\left(P_{k}\right)\right]^{T} . P_{k}$ denotes the number of spatial streams sent by UT $k$. The sequence $\left\{\mathbf{s}_{k}^{l}(p)\right\}_{l=1}^{L}$ is the DFT of $\left\{\mathbf{d}_{k}^{m}(p)\right\}_{m=1}^{L}$, where $\mathbf{d}_{k}^{m}=\left[\mathbf{d}_{k}^{m}(1)\right.$, $\left.\ldots, \mathbf{d}_{k}^{m}(p), \ldots, \mathbf{d}_{k}^{m}\left(P_{k}\right)\right]^{T}$ denotes the vector of data symbols sent by UT $k$ at subcarrier $m$ (with $\mathbb{E}\left[\left|\mathbf{d}_{k}^{m}(p)\right|^{2}\right]=\Sigma_{k}^{2}$ ). The data symbols are drawn from a $M$-QAM constellation.

\section{Backhaul Network (Signal Quantization)}

The $K$ small-cell APs are connected through a backhaul network to a $\mathrm{CU}$ allowing for the joint processing of the received signals, see Fig. 1. The total number of antennas associated to the CU is $T_{s}=T_{1}+\cdots+T_{K}$ and the total number of streams received is $P_{s}=P_{1}+\ldots+P_{k}$. It is assumed that the backhaul network has a limited capacity to convey the APs signals to the CU. We assume that at each AP the received signal is digitized, converted to the frequency domain and then quantized per subcarrier before being conveyed to the CU. The signal at each antenna is quantized independently of the other antennas. The quantized signal of antenna $t$

$$
\hat{\mathbf{y}}_{n}^{l}(t)=f_{Q}\left(\operatorname{Re}\left\{\mathbf{y}_{n}^{l}(t)\right\}\right)+j f_{Q}\left(\operatorname{Im}\left\{\mathbf{y}_{n}^{l}(t)\right\}\right),
$$

where $f_{Q}($.$) denotes a quantization function and the operators$ $\operatorname{Re}\{$.$\} and \operatorname{Im}\{$.$\} the real and imaginary part of \mathbf{y}_{n}^{l}(t)$. In this manuscript, for the sake of simplicity, we consider uniform quantizers, i.e. an uniform $f_{Q}($.$) has 2^{B}$ levels equally spaced between clipping levels $-A_{m}$ and $A_{m}$.

\section{Precoder AND EQualizers Design}

In this section, we jointly design the precoders and the iterative equalizers for both the macro and small-cells such that they can coexist under the same spectrum. The design is done under different levels of cooperation. First, we present the generic case where full-cooperation between the macro and small-cells is enabled. Then, we reduce the inter-system communication needs by proposing a limited information exchange approach for inter-system coexistence.

\section{A. Joint Precoder and Equalizer Design}

It is well known that for SC-FDMA based systems, linear equalization is not efficient to separate the spatial streams due to the residual inter-carrier interference [24]. In the context of heterogeneous systems, the equalizer should be designed to deal with both ICI and inter-system interferences. Therefore, a conventional linear equalizer design may not be the best strategy. To deal effectively with both the inter-system and inter-carrier interferences we jointly design an iterative frequency domain equalizer, based on IB-DFE principles, and a set of linear precoders at the small-cells. The macro-BS equalizer and the small-cell precoders are jointly designed so that the interference generation and cancelation procedures are optimally matched.

In the following, the index $k$ is dropped to improve the clarity of the equations as the presented derivation is for macro UT $(k=0)$.

1) Description of the iterative equalizer: A block diagram of the proposed IB-DFE received structure is depicted in Fig. 2. At the $i$ th iteration, the received signal on the $l$ th subcarrier, before the IDFT operation is given by

$$
\widetilde{\mathbf{s}}^{l, i}=\mathbf{F}^{l, i} \mathbf{y}_{B S}^{l}-\mathbf{B}^{l, i} \overline{\mathbf{s}}^{l, i-1},
$$

where $\overline{\mathbf{s}}^{l, i}=\left[\overline{\mathbf{s}}^{l, i}(1), \ldots, \overline{\mathbf{s}}^{l, i}(p), \ldots, \overline{\mathbf{s}}^{l, i}\left(M_{0}\right)\right]^{T} . \mathbf{F}^{l, i} \in$ $\mathbb{C}^{M_{0} \times T_{0}}$ denotes the feedforward matrix and $\mathbf{B}^{l, i} \in \mathbb{C}^{M_{0} \times M_{0}}$ the feedback matrix. The $L$-length sequence $\left\{\overline{\mathbf{s}}^{l, i}(p)\right\}_{l=1}^{L}$ is the 